\title{
Random Walks and Search in Time-Varying Networks
}

\author{
Nicola Perra, ${ }^{1}$ Andrea Baronchelli, ${ }^{1}$ Delia Mocanu, ${ }^{1}$ Bruno Gonçalves,${ }^{1}$ \\ Romualdo Pastor-Satorras, ${ }^{2}$ and Alessandro Vespignani ${ }^{1,3}$ \\ ${ }^{1}$ Laboratory for the Modeling of Biological and Socio-technical Systems, Northeastern University, Boston, Massachusetts 02115, USA \\ ${ }^{2}$ Departament de Física i Enginyeria Nuclear, Universitat Politècnica de Catalunya, Campus Nord B4, 08034 Barcelona, Spain \\ ${ }^{3}$ Institute for Scientific Interchange Foundation, Turin 10133, Italy
}

(Received 15 June 2012; published 4 December 2012)

\begin{abstract}
The random walk process underlies the description of a large number of real-world phenomena. Here we provide the study of random walk processes in time-varying networks in the regime of time-scale mixing, i.e., when the network connectivity pattern and the random walk process dynamics are unfolding on the same time scale. We consider a model for time-varying networks created from the activity potential of the nodes and derive solutions of the asymptotic behavior of random walks and the mean first passage time in undirected and directed networks. Our findings show striking differences with respect to the well-known results obtained in quenched and annealed networks, emphasizing the effects of dynamical connectivity patterns in the definition of proper strategies for search, retrieval, and diffusion processes in time-varying networks.
\end{abstract}

DOI: 10.1103/PhysRevLett.109.238701

PACS numbers: $89.75 . \mathrm{Hc}, 05.40 . \mathrm{Fb}$

Random walks on networks lie at the core of many realworld dynamical processes, ranging from the navigation and ranking of information networks to the spreading of diseases and the routing of information packets in largescale infrastructures such as the Internet [1-6]. In recent years, empirical evidence pointing out the heterogeneous topology of many real-world networks has led to a large body of work focusing on the properties of random walks in networks characterized by heavy-tailed degree distributions and other features such as clustering and community structure [1,7-12]. Although these studies provided a deeper understanding of processes of technological relevance such as World Wide Web navigation and ranking, they have mostly focused on the situation in which the time scale characterizing the changes in the structure of the network and the time scale describing the evolution of the process are well separated [13-17]. While convenient for analytical tractability, this limit is far from realistic in many systems including modern information networks, the diffusion and search of information in microblogging systems and social networking platforms, the spread of sexually transmitted diseases, or the diffusion of ideas and knowledge in social contexts. In all these cases, the concurrence of contacts and their dynamical patterns are typically characterized by a time scale comparable to that of the diffusion process, motivating the development of study models able to account for effects of the time-varying nature of networks on dynamical processes [18-27].

Motivated by the above problems, we study the random walk process in a fairly general class of time-varying networks. Namely, we consider the activity-driven class of models for time-varying networks presented in Ref. [23] that allows for an explicit representation of dynamical connectivity patterns. We derive the analytical solutions of the stationary state of the random walk and the mean first passage time [28] in both directed and undirected time-varying networks. We find that the behavior of the random walk and the ensuing network discovery process in time-varying networks is strikingly different from that occurring in quenched and annealed topologies [1,3,8,29]. These results have the potential to become a starting point for the definition of alternative strategies and mechanisms to explore and retrieve information from networks and more accurately characterize spreading and diffusion processes in a wide range of dynamic social networks.

Activity-driven network models focus on the activity pattern of each node, which is used to explicitly model the evolution of the connectivity pattern over time. Each node $i$ is characterized by a quenched fixed activity rate $a_{i}$, extracted from a distribution $F(a)$, that represents the probability per unit time that a given node will engage in an interaction and generate the corresponding edge connecting it with other nodes in the system. In the simplest formulation of the model, networks are generated according to the following memoryless stochastic process [23]: (i) At each time step $t$, the instantaneous network $G_{t}$ starts with $N$ disconnected nodes. (ii) With probability $a_{i} \Delta t$, each vertex $i$ becomes active and generates $m$ undirected links that are connected to $m$ other randomly selected vertices. Nonactive nodes can still receive connections from other active vertices. (iii) At time $t+\Delta t$, all the edges in the network $G_{t}$ are deleted and the process starts over again to generate the network $G_{t+\Delta t}$. It can be shown that the full dynamics of the network is encoded in the activity rate distribution, $F(a)$, and that the timeaggregated measurement of network connectivity yields a degree distribution that follows the same functional form 
as that of the distribution $F(a)$. This distribution can be assumed $a$ priori or derived from empirical data in the case of high-quality data sets such as those from social or information networks [23].

Although the above model is memoryless, it can be considered as the simplest yet nontrivial setting for the study of the concurrence of changes in connectivity pattern of the network and the dynamical processes unfolding on its structure. We define the random walk process on timevarying networks as follows: at each time step $t$ the network $G_{t}$ is generated, and the walker diffuses for a time $\Delta t$. After diffusion, at time $t+\Delta t$, a new network $G_{t+\Delta t}$ is generated (see Fig. 1). The concurrent dynamics of the random walker and the network thus take place with the same time scale, which introduces a feature not found in the equivalent processes in quenched or annealed networks, namely, that walkers can get trapped in temporarily isolated nodes. In other words, the diffusive dynamic of the particles is "enslaved" to the local connectivity pattern of each node so that effectively the diffusive process is transformed in a "transport" process defined concurrently by the network dynamic and the particle diffusive process.

The probability $P_{i}(t)$ that a random walker is in node $i$ at time $t$ obeys the master equation

$P_{i}(t+\Delta t)=P_{i}(t)\left[1-\sum_{j \neq i} \Pi_{i \rightarrow j}^{\Delta t}\right]+\sum_{j \neq i} P_{j}(t) \Pi_{j \rightarrow i}^{\Delta t}$,

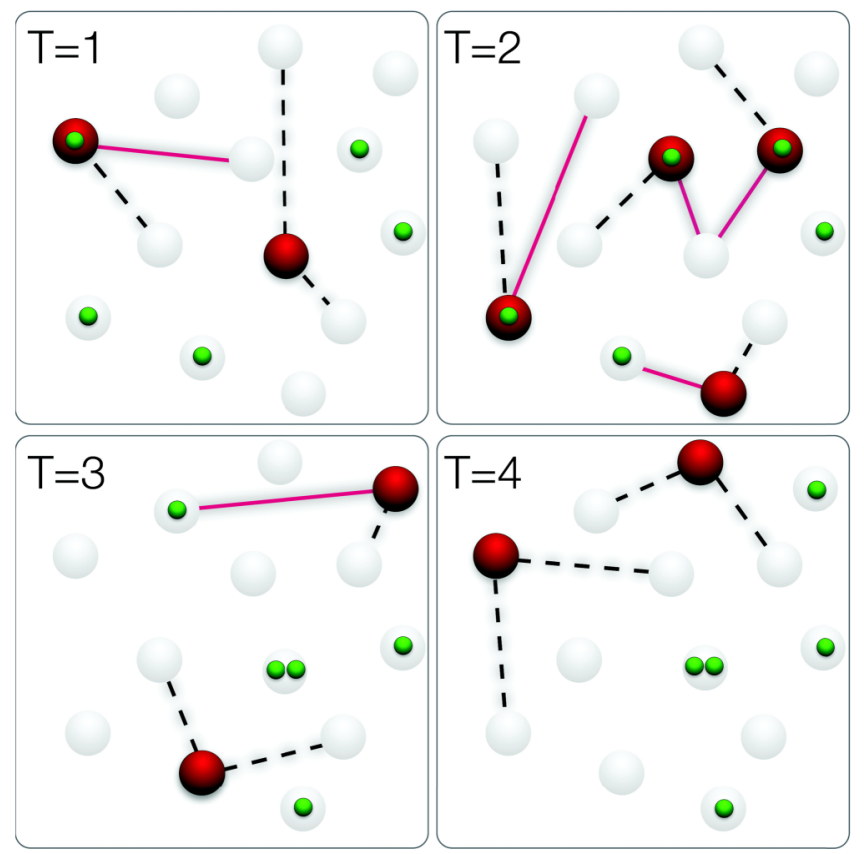

FIG. 1 (color online). Activity-driven random walk process. Active nodes are shown as fully colored (red) nodes. Walkers are presented as small (green) particles inside nodes. Links used by walkers to move from one node to another are shown in solid (red) lines, while edges connecting empty nodes are shown as dashed lines. Here we considered $m=2$. where $\Pi_{i \rightarrow j}^{\Delta t}$ is the propagator of the random walk, defined as the probability that the walker moves from vertex $i$ to vertex $j$ in a time interval $\Delta t$. At any time $t$, node $i$ will be linked to node $j$ if node $i$ becomes active and chooses to connect to node $j$ (with probability $m a_{i} \Delta t / N$ ) or if node $j$ becomes active and connects to node $i$ (with probability $\left.m a_{j} \Delta t / N\right)$. In the first case, the instantaneous average degree of node $i$, conditioned to the fact that it has become active, is $k_{i}=m(1+\langle a\rangle \Delta t)$, while in the second case we have $k_{i}=1+m\langle a\rangle \Delta t$, where the average is conditioned to the fact that a vertex $j$ has fired and has connected to $i$. A walker in node $i$ will then have to chose which one of the $k_{i}$ connections to follow. We focus on homogenous random walks. In this case, the probability of moving from node $i$ to node $j$ is inversely proportional to $k_{i}$. Thus the propagator can be written as

$$
\begin{aligned}
\Pi_{i \rightarrow j}^{\Delta t} & =\frac{m a_{i} \Delta t}{N} \frac{1}{m(1+\langle a\rangle \Delta t)}+\frac{m a_{j} \Delta t}{N} \frac{1}{1+m\langle a\rangle \Delta t} \\
& \simeq \frac{\Delta t}{N}\left(a_{i}+m a_{j}\right),
\end{aligned}
$$

where in the last expression we have neglected terms of order higher than $\Delta t$.

In order to obtain a system level description it is convenient to group nodes in the same activity class $a$, assuming that they are statistically equivalent, i.e., considering the limit $N \rightarrow \infty[1,6]$. Let us define the number of walkers in a given node of class $a$ at time $t$ as $W_{a}(t)=[N F(a)]^{-1}$ $W \sum_{i \in a} P_{i}(t)$, where $W$ is the total number of walkers in the systems. Considering Eq. (1) in the limit $\Delta t \rightarrow 0$ we can write

$$
\begin{aligned}
\frac{\partial W_{a}(t)}{\partial t}= & -a W_{a}(t)+a m w-m\langle a\rangle W_{a}(t) \\
& +\int a^{\prime} W_{a^{\prime}}(t) F\left(a^{\prime}\right) d a^{\prime},
\end{aligned}
$$

where $w \equiv W / N$ is the average density of walkers per node, and we have considered the continuous $a$ limit. The first two terms are contributions due to the activity of the nodes in class $a$, active nodes which release all the walkers they have and receive walkers originating from all the other nodes. The final two terms represent the contribution to inactive nodes due to the activity of the nodes in all the other classes. The stationary state of the process is

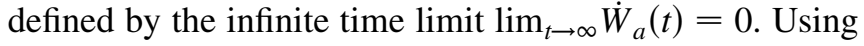
this condition in Eq. (3), we find the stationary solution

$$
W_{a}=\frac{a m w+\phi}{a+m\langle a\rangle},
$$

characterizing the stationary state of the random walk process in activity-driven networks, where $\phi=$ $\int a F(a) W_{a} d a$ is the average number of walkers moving out of active nodes. In the stationary state, this quantity is constant, and we can evaluate it self-consistently, which implies the equation 


$$
\phi=\int a F(a) \frac{a m w+\phi}{a+m\langle a\rangle} d a .
$$

By considering heavy-tailed activity distributions of the form $F(a) \sim a^{-\gamma}$, the explicit solution for $\phi$ can be written in terms of hypergeometric functions that can be numerically evaluated. Heavy-tailed activity distributions have been empirically measured in real-world time-varying networks [23].

To support the results of the analytical treatment, we have performed extensive Monte Carlo simulations of the random walk process in activity-driven networks with $N=$ $10^{5}$ nodes, $m=6$, and $w=10^{2}$ walkers. In particular, we consider a power-law distribution $F(a) \sim a^{-\gamma}$, with activity restricted in the interval $a \in[\epsilon, 1]$ to avoid possible divergencies in the limit $a \rightarrow 0$. As shown in Fig. 2, the analytical solution reproduces with great accuracy the simulation results. It is worth noting that in quenched and annealed networks the number of walkers in each node of degree $k$ is a linear function of the degree $W_{k} \sim k[1,8]$. However, in time-varying networks the number of walkers is not a linear function of the activity but saturates at large values of $a$. The difference is due to the properties of the instantaneous network, where the nodes with high activity have on average $k \sim m$ connections at each time step, and therefore a limited capacity for collecting walkers. This key feature cannot be recovered from time-aggregated views of dynamical networks. To clarify this question, we compare numerical simulations of walkers in a network obtained by integrating the activitydriven model with $N=10^{5}$ nodes, $m=6$, and $F(a) \sim a^{-2}$ over $T=50$ time steps with the results obtained in the instantaneous network (see inset of Fig. 2). The lack of a saturation is simply an artifact of using the timeaggregated network and highlights the importance of an appropriate consideration of the time-varying feature of

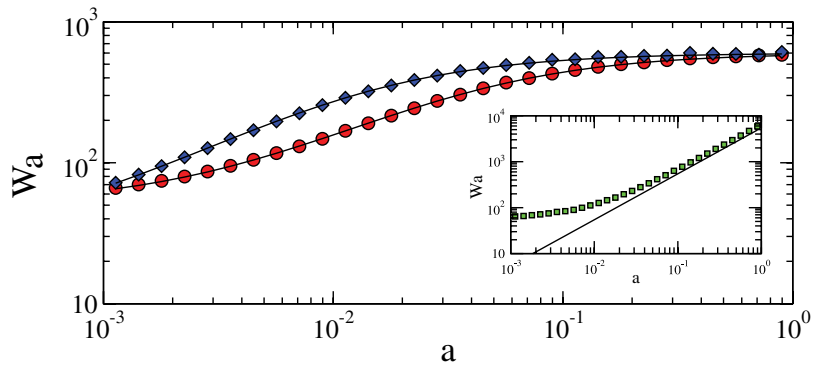

FIG. 2 (color online). Main plot: Stationary density $W_{a}$ of random walkers in activity-driven networks with activity distribution $F(a) \sim a^{-\gamma}$. We consider $\gamma=2$ (circles) and $\gamma=2.8$ (diamonds). Solid lines represent the analytical prediction of Eq. (4). Inset: Stationary density $W_{a}$ for random walks on top of an activity-driven network with $F(a) \sim a^{-2}$, integrated over $T=$ 50 time steps. The solid line corresponds to the curve $W_{a} \sim a$, fitting the simulation points for large values of $a$. Simulation parameters: $N=10^{5}, m=6, \epsilon=10^{-3}$, and $w=10^{2}$. Averages are performed over $10^{3}$ independent simulations. networks in the study of exploration and spreading processes in dynamical complex networks.

We next focus on the study of the transport dynamics in such networks by analyzing the mean first passage time (MFPT) [8,28], i.e., the average time needed for a walker to arrive at node $i$ starting from any other node in the network. In other words, the MFPT is the average number of steps needed to reach or find a specific target with obvious consequences for network discovery processes. Let us define $p(i, n)$ as the probability that the walker reaches the target node $i$ for the first time at time $t=$ $n \Delta t$. Since each node is able, in principle, to connect directly to any other node, this quantity is given simply by $p(i, n)=\xi_{i}\left(1-\xi_{i}\right)^{n-1}$, where $\xi_{i}$ is the probability that the random walker jumps to node $i$ in a time interval $\Delta t$. From Eq. (2), the probability that a walker in vertex $j$ jumps to $i$ in a time $\Delta t$ is given by $\Pi_{j \rightarrow i}^{\Delta t}$. Thus, we can write $\xi_{i}=\sum_{j}\left(W_{j} / W\right) \Pi_{j \rightarrow i}^{\Delta t}$, where we have replaced the probability that a single random walker is at node $j$ at time $t$ by its steady-state value $W_{j} / W$. The MFPT can thus be estimated as

$$
T_{i}=\sum_{n=0}^{\infty} \Delta t n p(i, n)=\frac{\Delta t}{\xi_{i}}=\frac{N W}{m a_{i} W+\sum_{j} a_{j} W_{j}} .
$$

Interestingly, the MFPT of each node $i$ is inversely proportional to its activity plus a constant contribution from all the other nodes, in clear contrast to what happens in quenched and annealed networks where $\xi_{i}$ is equivalent to the stationary state of the random walk, $\xi_{i}=W_{i} / W$. As before, the underlying cause of this fundamental difference is the fact that in activity-driven networks the walker can be trapped in a node with low activity for several time steps. The form of $\xi_{i}$ must then consider explicitly the dynamical connectivity patterns to account for the resulting delays. Figure 3 confirms these results with extensive Monte Carlo simulations matching the analytical results presented in Eq. (6).

The previous approach can be readily extended to the case of directed networks. By the use of the activity-driven framework, it is possible to define two types of timevarying directed networks. When a node $i$ is active, the $m$-generated links could be outgoing edges (Type I) or ingoing edges (Type II). For both types of directed networks it is possible to write down the diffusion propagator by following the same approach used in the undirected case. In particular, it is possible to show that if we define $W_{a}^{I}(t)$ and $W_{a}^{I I}(t)$ as the number of walkers in networks of Type I and II, respectively, their stationary values read as

$$
W_{a}^{I}=\frac{w}{a} \frac{1}{\left\langle\frac{1}{a}\right\rangle} ; \quad W_{a}^{I I}=a w \frac{1}{\langle a\rangle} .
$$

While we will report the full calculation elsewhere, this result can be intuitively understood by considering that in Type I networks active nodes create outgoing links. 


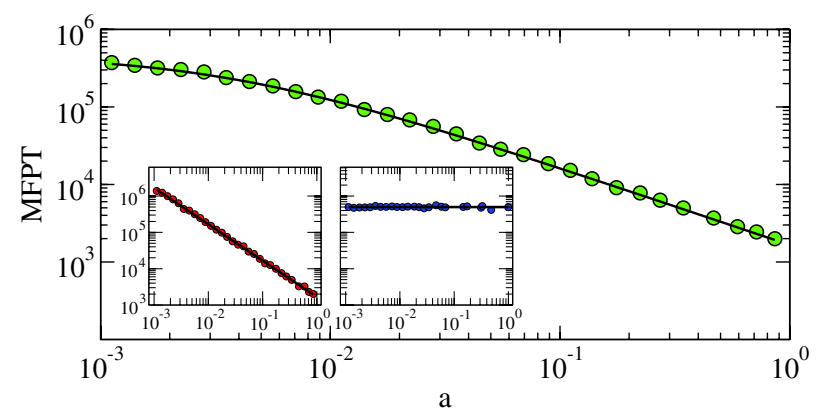

FIG. 3 (color online). Main plot: MFPT of a random walker as a function of the activity $a$ in activity-driven networks with activity distribution $F(a) \sim a^{-2}$. Full line corresponds to the theoretical prediction of Eq. (6). Right inset: MFPT as a function of activity for directed Type I activity-driven networks. Left inset: MFPT as a function of activity for directed Type II activity-driven networks. Simulation parameters: $N=10^{4}$ ( $N=10^{3}$ for Type I, blue dots in right inset), $m=6$, and $\epsilon=10^{-3}$. Averages are performed over $10^{3}$ independent simulations for each activity class.

Walkers are thus more likely to diffuse out of these nodes, meaning that the higher the activity, the smaller the number of walkers in the nodes of that class. In Type II networks, on the other hand, active nodes create ingoing links. Walkers are thus more likely to diffuse into high activity nodes, and the scaling of the stationary state is linear with the activity. Interestingly, the undirected functional form of the stationary state is a combination of these two different behaviors.

By following the same reasoning used for the undirected case, it is straightforward to derive the analytic expression of the MFTP for directed activity networks, namely,

$$
T_{i}^{I}=\frac{N W}{\sum_{j} a_{j} W_{j}} ; \quad T_{i}^{I I}=\frac{N}{m a_{i}}
$$

In the first case, the MFPT is independent of the activity of the considered node. The walker can move to node $i$ just when other active nodes create outgoing links pointing to $i$. In the second case, the MFPT is just proportional to the activity of the node $i$ and is not a function of the activity of the other nodes. In this case we also recover that the propagator of the random walk for undirected activitydriven networks has these two symmetric contributions that both contribute to the undirected MFPT.

The analytical results can be validated by means of Monte Carlo simulations. The right inset of Fig. 3 refers to activity networks of Type I. We fix $N=10^{3}, m=6$, one walker, and activities distributed according to a power-law $F(a) \sim a^{-2}$. We then measured the MFPT selecting $10^{3}$ targets for each activity class. The simulations are indistinguishable from the analytical result in Eq. (8). The left inset in Fig. 3 refers to activity networks of Type II under the same simulation parameters except for the number of nodes fixed to $N=10^{4}$ in this case. Again, a perfect match is observed with the analytical result Eq. (8).

From the above results, it is evident that the dynamics of time-varying networks significantly alters the standard picture achieved for dynamical processes in static networks. Focusing on the specific case of activity-driven networks and the simple random walk process, the present results open the path to a number of future studies where the dynamics of the network will have to be considered to avoid misleading results in the analysis of dynamical processes in most situations of practical interest. Finally, we note that the time-varying networks model we have considered is Markovian (memoryless) and lacks dynamical correlations, at odds with many real-world dynamical networks [21]. The investigation of the effects of these relevant properties on diffusion calls for additional research efforts.

The work has been partly sponsored by the Army Research Laboratory and was accomplished under Cooperative Agreement No. W911NF-09-2-0053. R. P.-S. acknowledges financial support from the Spanish MICINN (FEDER), under Project No. FIS2010-21781-C02-01, and ICREA Academia, funded by the Generalitat de Catalunya.

[1] A. Barrat, M. Barthélemy, and A. Vespignani, Dynamical Processes on Complex Networks (Cambridge University Press, Cambridge, England, 2008).

[2] R. Cohen and S. Havlin, Complex Networks: Structure, Robustness and Function (Cambridge University Press, Cambridge, England, 2010).

[3] M. Newman, Networks. An Introduction (Oxford University, New York, 2010).

[4] R. Albert and A.-L. Barabási, Rev. Mod. Phys. 74, 47 (2002).

[5] S. Boccaletti, V. Latora, Y. Moreno, M. Chavez, and D.-U. Hwang, Phys. Rep. 424, 175 (2006).

[6] A. Vespignani, Nat. Phys. 8, 32 (2012).

[7] J. Kleinberg, Nature (London) 406, 845 (2000).

[8] J.D. Noh and H. Rieger, Phys. Rev. Lett. 92, 118701 (2004).

[9] L. K. Gallos, Phys. Rev. E 70, 046116 (2004).

[10] B. Kozma, M. B. Hastings, and G. Korniss, Phys. Rev. Lett. 95, 018701 (2005).

[11] A. N. Samukhin, S. N. Dorogovtsev, and J. F. F. Mendes, Phys. Rev. E 77, 036115 (2008).

[12] A. Baronchelli, M. Catanzaro, and R. Pastor-Satorras, Phys. Rev. E 78, 011114 (2008).

[13] S. Brin and L. Page, Computer Networks ISDN Systems 30, 107 (1998).

[14] J. Kleinberg, J. Assoc. Comput. Mach. 46, 604 (1999).

[15] L. A. Adamic, R. M. Lukose, A. R. Puniyani, and B. A. Huberman, Phys. Rev. E 64, 046135 (2001).

[16] D. J. Watts, P.S. Dodds, and M.E. J. Newman, Science 296, 1302 (2002).

[17] J. Kleinberg, in Proceedings of the International Congress of Mathematicians (European Mathematical Society, Zurich, 2006). 
[18] M. Morris, Nature (London) 365, 437 (1993).

[19] E. Volz and L. A. Meyers, J. R. Soc. Interface 6, 233 (2009).

[20] L. B. Shaw and I. B. Schwartz, Phys. Rev. E 81, 046120 (2010).

[21] P. Holme and J. Saramäki, Phys. Rep. 519, 97 (2012).

[22] S. Jolad, W. Liu, B. Schmittmann, and R. K. P. Zia, arXiv:1109.5440.

[23] N. Perra, B. Gonçalves, R. Pastor-Satorras, and A. Vespignani, Sci. Rep. 2, 469 (2012).

[24] M. Starnini, A. Baronchelli, A. Barrat, and R. PastorSatorras, Phys. Rev. E 85, 056115 (2012).
[25] P. Basu, S. Guha, A. Swami, and D. Towsley, in Fourth International Conference on Communication Systems and Networks, COMSNETS 2012, Bangalore, India, January 3-7, 2012, edited by K. K. Ramakrishnan, R. Shorey, and D. F. Towsley (IEEE, 2012).

[26] Z. Toroczkai and H. Guclu, Physica (Amsterdam) 378A, 68 (2007).

[27] C. T. Butts, Socio. Meth. 38, 155 (2008).

[28] S. Redner, A Guide To First-Passage Processes (Cambridge University Press, Cambridge, England, 2001).

[29] A. Baronchelli and R. Pastor-Satorras, Phys. Rev. E 82, 011111 (2010). 